\title{
Fonología de la frase entonativa
}

\author{
Phonology of the intonational phrase \\ Guillermo Toledo \\ Université Laval, Canadá y Conicet, Argentina. \\ e-mail: gatoledo@onicet.gov.ar
}

\begin{abstract}
Se estudia la asociación fonológica primaria y secundaria en el contraste de continuidad textual opuesto a conclusión. Este problema se investiga en el contexto de la frase entonativa intermedia, menor (ip) y la frase entonativa mayor (IP), en el acento de la frase (el ip $\mathrm{H}-$ ) y en el acento de la frase y el tono de frontera (ip L- IP L\%). Los acentos tonales finales de frases pertenecen a palabras proparoxítonas, paroxítonas y oxítonas. El corpus se integra por textos leídos emitidos por su autor, un informante de Colombia. Los resultados indican que el acento de la frase y el tono de frontera influyen en la asociación fonológica con el propósito de marcar el contraste pragmático entre las dos frases opuestas: la frase menor (marca de continuidad) y la frase mayor (marca de conclusión).
\end{abstract}

Palabras clave: fraseo entonativo en español, asociación fonológica primaria y secundaria, contraste lingüístico, contraste semántico y pragmático.

The primary and secondary phonological associations in the contrast of textual continuity versus conclusion are studied. This problem is investigated in the context of the intonational intermediate phrase, minor (ip), and the intonational major phrase (IP), in the phrase accent (ip H-) and in the phrase accent and the edge tone (ip L- IP L\%). The final pitch accents of phrases belong to proparoxytone, paroxytone and oxytone words. The corpus is integrated by texts read and emitted by the author, an informant from Colombia. The results indicate that the phrase accent and edge tone influence the final tonal accent through changes in the phonological association, with the purpose of marking the pragmatic contrast among the two opposite phrases: the minor phrase (continuity) and the major phrase (conclusion).

Key words: Spanish phrasing, primary and secondary phonological association, linguistic contrast, semantic and pragmatic contrast.

\section{INTRODUCCIÓN}

1.1. Modelo métrico y autosegmental. En el modelo métrico y autosegmental (AM) se asocian dos cadenas sintagmáticas: el texto y el tono (Ladd 1996: 42; Hualde 2003). Las dos cadenas son independientes, pero se vinculan sólo en ciertos puntos, en las sílabas acentuadas (Pierrehumbert 1980; Pierrehumbert y Beckman 1988; Ladd 1996; Gussenhoven 2002; Beckman et al. 2002; Beckman et al. 2005). Desde un punto de vista métrico, las sílabas acentuadas son fuertes y los segmentos inacentuados son débiles. Los tonos son fuertes en las sílabas acentuadas. Los tonos en los segmentos 
inacentuados son transicionales: concatenan los acentos tonales al contorno de entonación. En cada acento tonal se actualizan uno o dos tonos: uno, central, alineado con la duración de la acentuada y otro, periférico, en la sílaba pretónica o en el inicio de la sílaba acentuada o en la sílaba postónica (Pierrehumbert 1980: 25; Ladd 1996: 79). La centralidad del tono en el acento tonal se indica por el diacrítico estrella: H*, L* (Ladd 1996: 79). Los acentos tonales son targets 'blancos' estáticos, no son inflexiones dinámicas, y son relativos con respecto a un rango tonal del hablante (el punto relativamente más bajo o el punto relativamente más alto). El contraste es paradigmática, según el rango tonal y no en forma sintagmática de acuerdo al nivel tonal de otro acento adyacente (Ladd 1996: 61; Beckman et al. 2005). En la secuencia LH (bajo, alto) o en la secuencia HL (alto, bajo), en el desarrollo temporal de la sílaba acentuada y en el contexto pretónico y postónico, se producen diferentes alineaciones fonéticas y, por ello, también se producen distintas asociaciones fonológicas. Este nivel formal de los acentos tonales constituye la taxonomía propia de cada dialecto dentro de cada lengua: indica el contraste lingüístico, específicamente, las relaciones pragmáticas (Hualde 2003). Para el español, el análisis formal da como resultante dos acentos tonales monotonales $\left(\mathrm{H}^{*}, \mathrm{~L}^{*}\right)$ y cuatro acentos bitonales $\left(\mathrm{L}^{*}+\mathrm{H}, \mathrm{L}+\mathrm{H}^{*}\right.$, $\mathrm{H}+\mathrm{L}^{*}, \mathrm{H}^{*}+\mathrm{L}$ ); ver el esquema en Fig. 1. Como se explica antes, la marca estrella indica el tono dentro del marco de la sílaba acentuada, el tono central. La ausencia de esta marca muestra el tono periférico y débil, fuera del marco de la sílaba acentuada, la pretónica (pret) y la postónica (post) (Hualde 2003).

Figura 1

Acentos tonales

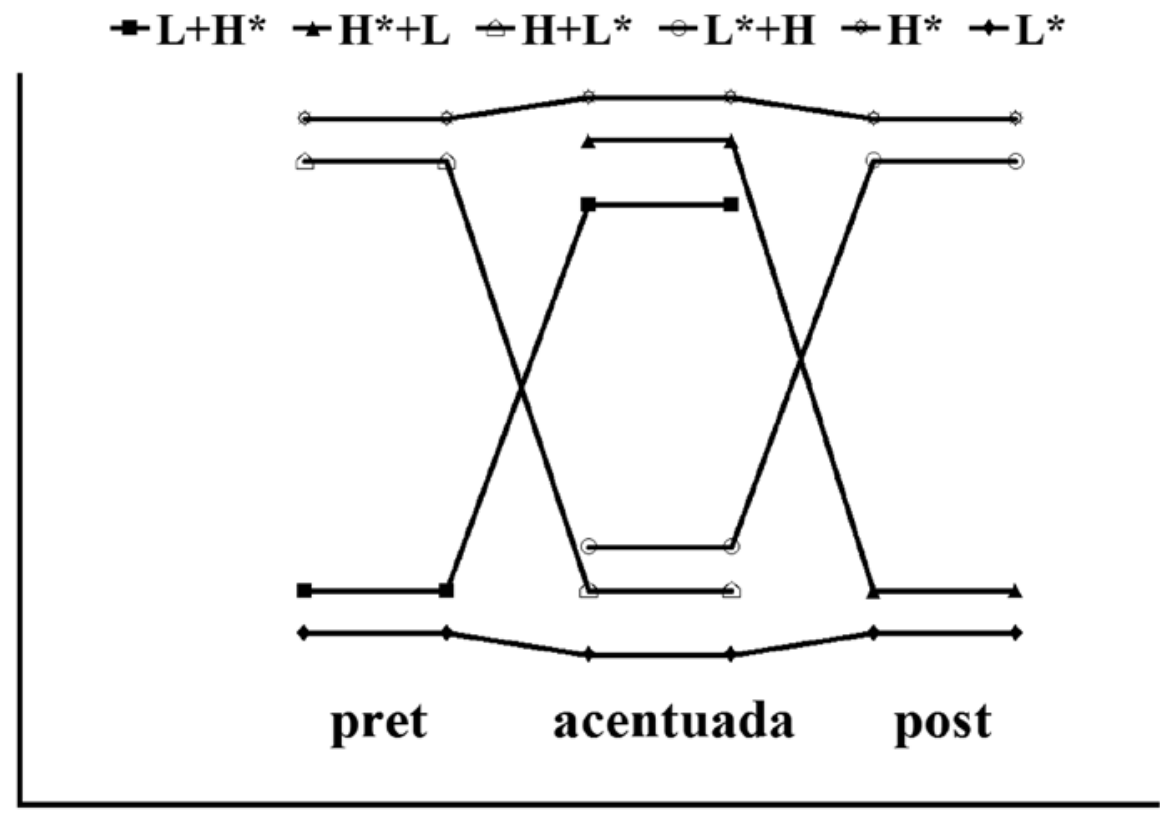


Tabla 1

Acentos tonales y tonemas producidos por hablantes hispanoamericanos (adaptado de Sosa 1999: 187-197), emisión declarativa Le dieron el número de vuelo

\begin{tabular}{|l|l|l|l|l|}
\hline Bogotá & $\mathrm{L}^{*+\mathrm{H}}$ & $\mathrm{L}^{*}+\mathrm{H}$ & $\mathrm{H}^{*}$ & $\mathrm{~L} \%$ \\
México & $\mathrm{L}^{*+\mathrm{H}}$ & $\mathrm{H}^{*}$ & $\mathrm{~L}^{*} \mathrm{H}^{*}$ & $\mathrm{~L} \%$ \\
San Juan & $\mathrm{L}^{*+\mathrm{H}}$ & $\mathrm{L}^{*+\mathrm{H}}$ & $\mathrm{L}^{*}$ & $\mathrm{~L} \%$ \\
Caracas & $\mathrm{L}^{*+\mathrm{H}}$ & $\mathrm{L}^{*}+\mathrm{H}$ & $\mathrm{H}^{*}$ & $\mathrm{~L} \%$ \\
Cuba & $\mathrm{L}^{*}+\mathrm{H}$ & $\mathrm{L}^{*}+\mathrm{H}$ & $\mathrm{L}^{*}$ & $\mathrm{~L} \%$ \\
Buenos Aires & $\mathrm{H}^{*+\mathrm{L}}$ & $\mathrm{H}^{*}$ & $\mathrm{~L}^{*}$ & $\mathrm{~L} \%$ \\
\hline
\end{tabular}

Sosa (1999: 188-189) presenta una taxonomía para el español de Colombia que sigue los patrones tonales básicos del español panhispánico. Los enunciados declarativos presentan acentos prenucleares (el pretonema) $\mathrm{L}^{*}+\mathrm{H}$ y un acento nuclear $\mathrm{H}^{*}$. Se suma el acento de frontera final L\% (Confróntese con otras emisiones de hablantes hispanoamericanos: Tabla I). Asimismo, en el español bogotano, las oraciones interrogativas absolutas muestran un pretonema con acentos tonales $\mathrm{L}^{*}+\mathrm{H}$ y un acento nuclear L*. El tono de frontera es, naturalmente, H\% (Sosa 1999: 199-200). Sin embargo, Sosa (1991) analiza oraciones interrogativas absolutas del español bogotano y obtiene acentos prenucleares $\mathrm{H}^{*}, \mathrm{H}+\mathrm{H}^{*}$ y $\mathrm{H}^{*}+\mathrm{H}$. Los tonos de frontera fluctúan entre L\% y H\%. Hay diferencias significativas entre Sosa (1991) y Sosa (1999). En el primer estudio se registran un número de casos con una asociación fonológica entre el tono y la sílaba acentuada ( $\mathrm{H}^{*}$ y tonos periféricos posibles). En el segundo estudio, los acentos prenucleares se caracterizan por una asociación entre el tono y la sílaba acentuada con un nivel bajo $\left(\mathrm{L}^{*}\right)$; en cambio, el tono periférico (en la postónica) tiene un nivel alto $(\mathrm{H})$. El primitivo fonológico es generalmente $\mathrm{L}^{*}+\mathrm{H}$.

1.2. Fraseo entonativo y fronteras. Existen dos criterios netos con respecto a las unidades de entonación que estructuran el fraseo entonativo del español: un fraseo basado en una única unidad (Sosa 1999; Beckman et al. 2002; Jun 2005) y un fraseo entonativo basado en dos unidades (Nibert 2000; Face; 2001; Hualde 2003; Barjam 2004; Alvord 2006; Toledo y Ramírez Verdugo 2007). Sosa (1999) propone una frase entonativa mayor (IP). Es un grupo entonativo que puede estructurar la oración como único constituyente. Está constituido por uno o más acentos tonales y un tono de frontera que cierra la unidad (T\%). Este tono de frontera puede ser $\mathrm{L} \%$ o $\mathrm{H} \%$. El hablante decide sobre emisiones mayores de acuerdo a fenómenos de reestructuración, por segmentación de los fragmentos de discurso por medio de nuevas IP: [nos contaron la triste historia de un ranchero enamorado] IP, [nos contaron] IP [la triste historia de un ranchero enamorado] IP, [nos contaron] IP [la triste historia] IP [de un ranchero enamorado] IP (Sosa 1999: 43). Von Heusinger (2007) describe esta frase entonativa mayor del siguiente modo: (1) la unidad puede producirse entre pausas; (2) este constituyente termina con una frontera tonal; (3) estas unidades no son isomórficas con la estructuración sintáctica, pero las dos estructuras se unen convenientemente; (4) esta frase entonativa completa una unidad de sentido. Según el Sp-ToBI (el inventario de tonos y fronteras del español), la unidad se produce con un índice de corte con un nivel 4, el grado de corte acústico y perceptivo más drástico del fraseo entonativo (Beckman et al. 2002; Beckman et al. 2005). La otra posición propone dos unidades entonativas y jerarquizadas (Nibert, 2000; Face; 2001; Hualde 
2003; Barjam 2004; Alvord 2006; Toledo y Ramírez Verdugo 2007). La frase entonativa mayor (IP) es el continente de frases entonativas intermedias (ip). Esta frase intermedia es similar a la mayor (IP), pero las características prosódicas son menos relevantes. Nibert (2000) describe la frase entonativa intermedia en el español, la presenta del siguiente modo: (1) la unidad muestra un cambio tonal en la zona derecha de su desarrollo, en el contexto de la frontera; (2) tiene un alargamiento temporalo de la sílaba final antes de esa frontera; (3) presenta un índice de corte de nivel 3 (según el Sp-ToBI, Beckman et al., 2002 y Beckman et al., 2005); (4) un cambio tonal en la próxima ip: una inflexión descendente, un contorno de poca pendiente o plano. La estructura es [(ip) T- T\% IP]. El T- es el acento de frase o el tono de frontera de la frase intermedia (H- o L-). Se ejemplifica del modo siguiente: [(el camión) ip H- (se despeñó sobre Gijón) ip L- IP L\%]. En este caso específico, la primera frase intermedia constituye la rama tensiva de la emisión declarativa (la prótasis) y la segunda frase intermedia es la rama distensiva (la apódosis) (ver Figura 2). De esta manera Face (2001) analiza el español de Madrid. Así Barjam (2004) estudia la entonación del dialecto de Buenos Aires. Asimismo, Alvord (2006) presenta sus resultados sobre el español hablado en Miami. De forma similar, Toledo y Ramírez Verdugo (2007) presentan hallazgos sobre habla de laboratorio emitida por hablantes de Barcelona, Alicante y Buenos Aires.

1.3. Asociación fonológica secundaria. El anclaje fonológico entre las dos cadenas sintagmáticas, el texto y el tono, se produce en las sílabas fuertes, las acentuadas. La asociación fonológica es primaria e indica el nivel subyacente de los acentos tonales como primitivos fonológicos. Esta asociación se produce en un único nivel jerárquico, en la sílaba acentuada. En cambio, la asociación fonológica secundaria se produce en niveles jerárquicos distintos. El tono se ancla también a acentos de frase y a tonos de juntura; de este modo, los tonos sufren la influencia de estos constituyentes jerárquicos superiores a la sílaba acentuada. De esta manera, se decriben estas asociaciones en el trabajo pionero de Pierrehumbert y Beckman (1988). Posteriormente, Grice (1995) utiliza la asociación secundaria de tonos que se anclan a frases entonativas intermedias; obtiene esos hallazgos en muestras de italiano, en el dialecto de Palermo.

El objetivo de este trabajo es el estudio de las frases entonativas menores y mayores en un corpus leído. Se analizan las diferencias provocadas por los acentos tonales finales de frase dentro de palabras proparoxítonas, paroxítonas y oxítonas confrontados a los tonos de frontera de esas frases. Se estudia la asociación de los tonos a constituyentes de mayor nivel jeráquico dentro del fraseo fonológico. Por último, se profundiza el contraste lingüístico, pragmático, esto es, la continuidad discursiva opuesta a la conclusión discursiva. Esta es la estructura del trabajo. En el Apartado 1 se presenta una introducción al tema integrada por varias Secciones. En la Seccción 1 se explica el modelo métrico y autosegmental (AM) y los acentos tonales de acuerdo a varias propuestas, entre otras, la propuesta de Hualde (2003). En la Sección 2 se comenta el fraseo fonológico de la entonación: las frases entonativas intermedias, menores (ip), y las frases entonativas mayores (IP); asimismo, se presentan los tonos de frontera intermedia, el acento de frase (T-) y el tono de frontera mayor (T\%). En la Sección 3 se desarrolla la asociación fonológica primaria entre la sílaba acentuada y el tono, en un único constituyente: la sílaba acentuada. Esta aclaración es la base para la revisión de la asociación fonológica secundaria. Aquí se vincula el tono con 
Figura 2

Frase entonativa intermedia (ip), acento de frase (H-), rama tensiva, hablante de Buenos Aires

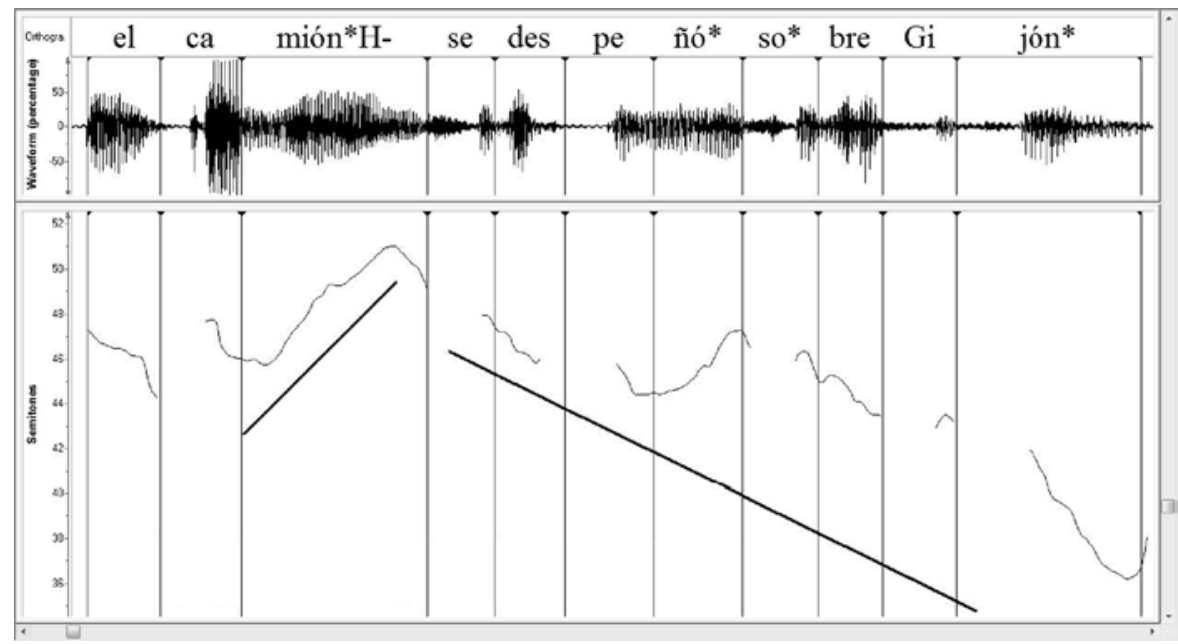

un nivel superior de la jerarquía fonológica: las frases entonativas. En el Apartado 2 se puede observar la metodología del trabajo, en dos Secciones. En la Sección 1 se presenta el corpus de textos leídos y el dialecto del informante, el lector. En la Sección 2 se explica el análisis acústico del material seleccionado. En el Apartado 3 se analizan las frases entonativas menores y mayores, los acentos tonales y los problemas de asociación fonológica primaria y secundaria registrados. En el Apartado 4 se presenta la conclusión y se discuten los resultados obtenidos por medio de argumentaciones generales sobre el modelo AM de la entonación y por medio de la confrontación con otros trabajos realizados sobre este tema.

\section{Metodología}

2.1. El corpus estudiado. Se estudia un corpus literario emitido por su autor, Gabriel García Márquez. El dialecto es el español colombiano (Gabriel García Márquez, Palabras de esta América, fragmentos de El amor en los tiempos del cólera, 1997, Casa de las Américas, La Habana, Cuba). Los materiales tienen una duración de 43 minutos. Se analizan 180 oraciones complejas. Las oraciones son de este tipo: [(Fue por esa época) ip (cuando Florentino Ariza decidió contarle en sus cartas a Fermina Daza) ip (que estaba empeñado en rescatar para ella el tesoro del galeón sumergido.) ip] IP. Se segmentan las frases entonativas intermedias y la frase entonativa final (las barras verticales indican los segmentos finales de las frases, en la Figura 3). Se analizan los tonemas: el acento tonal final de la ip y el acento de frase T-, el acento nuclear y el tono de frontera de IP (ver la Fig. 3). Se seleccionan 72 frases entonativas intermedias con acento de frase H-, también se consideran para el estudio 20 frases intermedias con el acento tonal final en palabras proparoxítonas. En estas últimas, la sílaba acentuada y el tono periférico se separa del acento de frase. En los acentos 
tonales finales en palabras paroxítonas, la sílaba acentuada se separa del acento de frase, pero no así el tono periférico. En los acentos tonales finales en palabras oxítonas, la sílaba acentuada se incrusta en el acento de frase H-.

Existe un contraste lingüístico entre las frases entonativas menores (ip) y mayores (IP); hay diferencias semánticas y pragmáticas, discursivas, entre las dos frases. La frase intermedia con tono de frontera $\mathrm{H}$ - indica que esa frase tiene cohesión semántica $\mathrm{y}$, esencialmente, continuidad discursiva con las siguientes frases que integran la frase entonativa mayor (IP); en cambio, esta frase IP tiene marca de conclusión, básicamente en su tono de frontera L\% (Pierrehumbert y Hirschberg, 1986; Pierrehumbert y Hirschberg 1990; Prevost 1995: 48). En las emisiones interrogativas se produce un contraste similar, aunque no es el motivo de este estudio.

Figura 3

Acentos tonales finales de ip, acentos de frase, acento nuclear de IP y tono de frontera

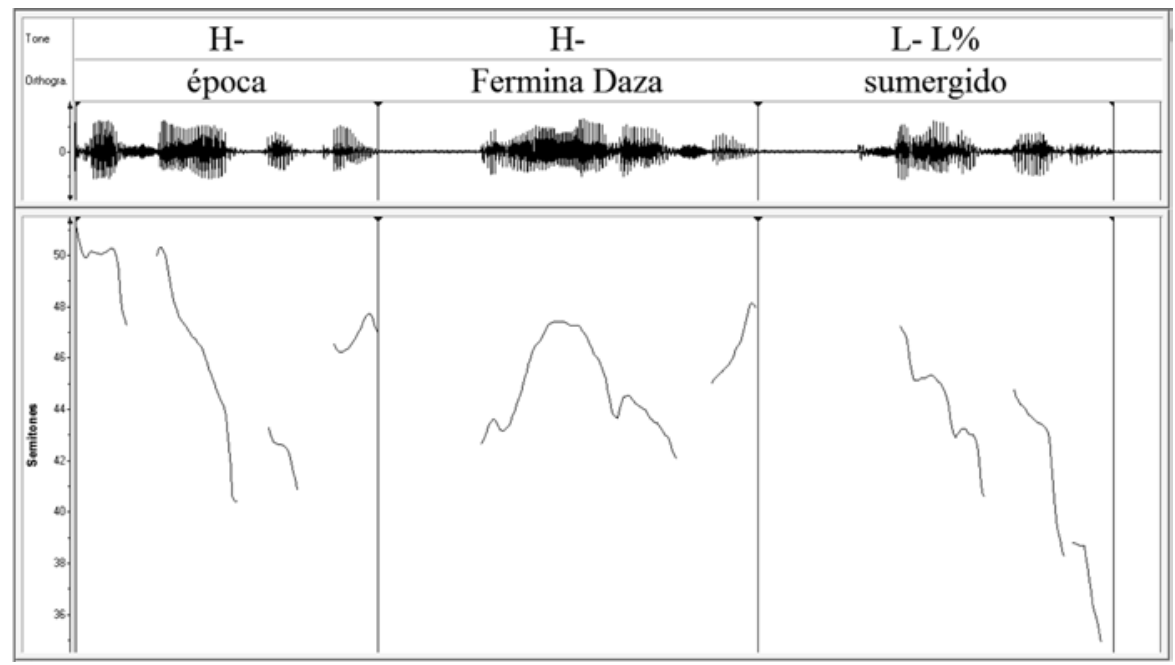

2.2. Análisis acústico. Se analizan los contornos de entonación por medio del programa de computación Speech Analyzer 3.0 (2007, Summey Institute of Linguistics). Las Figuras también se realizan con el mismo programa. En la parte superior de ellas, se presenta la forma de onda; en la parte inferior se puede observar el contorno de entonación. El eje vertical en esta parte inferior está seccionado en dos semitonos, un valor estándar para este programa. La Figura está dividida en barras verticales que segmentan las sílabas. Existe un caso especial: la Fig. 3 está dividida en barras verticales que separan los segmentos finales de las tres frases entonativas intermedias y la frase entonativa mayor. En la parte superior de la Figura se indican las transcripciones tonales y ortográficas.

\section{ANÁLISIS DE LAS FRASES ENTONATIVAS}

3.1. Acentos tonales en palabras proparoxítonas, paroxítonas y oxítonas. En la Fig. 4 se muestra una fragmento de una frase entonativa intermedia (ip). Pertenece a la 
Figura 4

Fragmento de una ip, acento tonal final, acento de frase

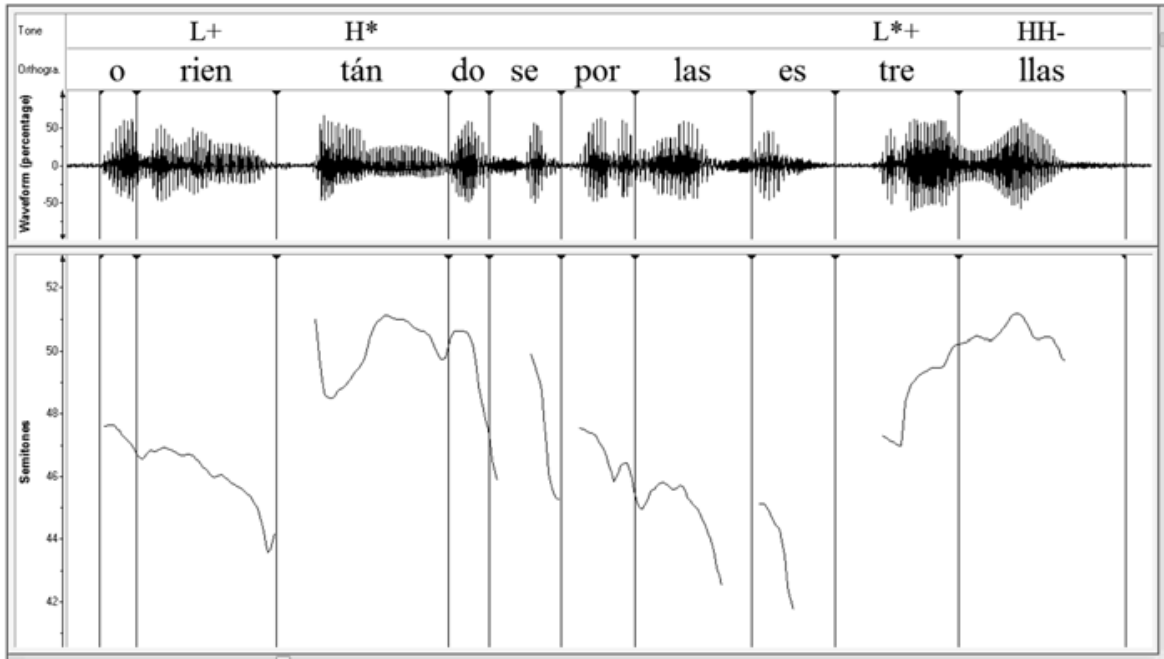

oración siguiente: [(Le preguntó si era capaz de navegar de noche orientándose por las estrellas) ip, (y Euclides le dijo que sí) ip] IP. La Figura registra dos acentos tonales de la ip: el primer acento tonal es oriéntandose y el acento final de la ip es estrellas. El primer acento presenta un valle en la sílaba pretónica (L) y el pico se desarrolla a lo largo de la sílaba acentuada. La asociación primaria vincula el tono con esa sílaba acentuada (ver la Fig. 1 y Hualde 2003). Este acento tonal no sigue las descripciones estándares del español panhispánico. En esas descripciones, los acentos prenucleares son $\mathrm{L}^{*}+\mathrm{H}$, es decir, con un valle y un recorrido tonal sobre la sílaba acentuada y el pico supera esa sílaba y culmina en la sílaba postónica (Sosa 1999; Face 2001; Hualde 2003; Face y Prieto 2007). En cambio, el acento tonal final estrellas es L*+H, es decir, el pico tonal supera la sílaba acentuada y culmina en la sílaba postónica. Se produce una asociación primaria entre el pico y la sílaba acentuada (L). El tono periférico recibe el pico en el espacio tonal de la sílaba postónica. El anclaje del tono se asocia al acento de frase, es H H-. La asociación se produce en un nivel jerárquico superior, en el nivel de la frase entonativa intermedia. Asimismo, la marca indica la continuidad textual o discursiva.

En la Fig. 5 se observa un fragmento de una ip y la IP final de la oración consignada en en el texto de la Fig. 4. Nuevamente, no se registran acentos prenucleares estándares según la descripción del español panhispánico (Euclides y dijo). Sosa (1999: 140) indica que el español se caracteriza por un pretonema rebotado, es decir, la sílabas acentuadas son bajas (L) y crecen en la sílaba postónica. El término rebotado es una metáfora de una pelota de tenis que golpea en la sílabas acentuadas y rebota en las sílabas postónicas; sin embargo, cada vez el rebote es menos intenso, el fenómeno del escalonamiento descendente de la emisión declarativa. Ese esquema no funciona en este texto leído, por lo menos en los casos registrados hasta ahora. Los acentos son $\mathrm{L}+\mathrm{H}^{*}$, tienen un valle en la sílaba pretónica y un crecimiento que culmina en la sílaba acentuada, la sílaba postónica es más baja. El último acento (sí), tiene una 
inflexión descendente hacia el nivel bajo del infromante, es $\mathrm{L}^{*}$. El acento de frase y el tono de frontera es L- L\%. La frase ip y la IP marcan la conclusión textual o discursiva. Se produce el contraste con las frases intermedias, que son continuativas. Sosa (1999: 188) registra en español bogotano algunos casos de acentos tonales $\mathrm{H}^{*}$ L\%, naturalmente, en el tonema de algunas emisiones declarativas. En su análisis no existen acentos de frase.

Figura 5

Fragmento de una ip y la IP, acento fonal final, acento de frase, tono de frontera

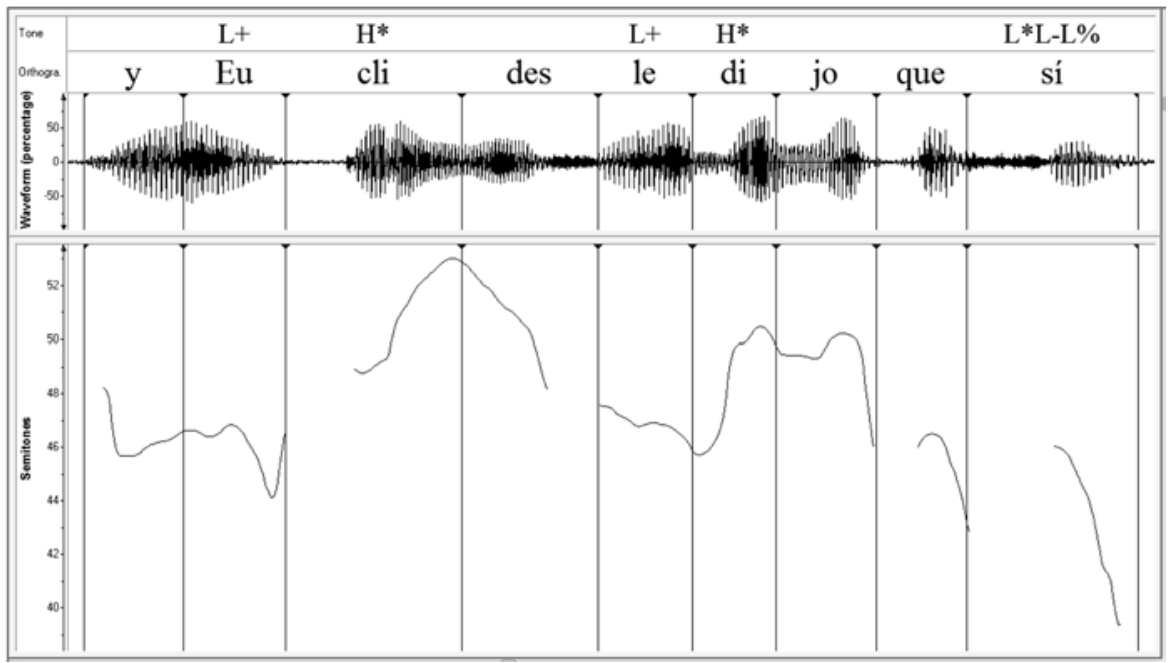

En la Fig. 6 se observan tres frases entonativas intermedias (ip) dentro de una frase mayor, final (IP). Pertenecen a la siguiente oración del texto: [(Le preguntó si sabía defenderse de los tiburones) ip, (y Euclides le dijo que sí,) ip (pues tenía artificios mágicos para espantarlos.) ip IP]. Se muestran los acentos tonales finales y el acento de frase (H-), en la tercera ip, se puede observar el acento tonal final, el acento de frase y el tono de frontera $(\mathrm{L}-\mathrm{L} \%)$. El primer acento tonal final presenta un valle en la sílaba pretónica, se desarrolla tonalmente a lo largo de la sílaba acentuada y culmina en la sílaba postónica. El acento tonal es $\mathrm{L}^{*}+\mathrm{H}$ (ver la Fig. 1 y Hualde 2003). Este acento tonal, dentro de una palabra paroxítona presenta una asociación primaria entre el tono y la sílaba acentuada ( $\mathrm{L}^{*}$ ) y una asociación fonológica secundaria de ese tono con el acento de frase (HH-). La presencia del acento de frase provoca un cambio en el acento tonal: el pico se produce dentro de la sílaba postónica. El tipo de palabra en donde se actualiza el acento tonal, una palabra paroxítona, con una única sílaba postónica, coadyuva en esa asociación fonológica secundaria. El tono periférico, en la sílaba postónica, se inserta en el espacio del acento de frase. El resultado es H H-. En el segundo acento tonal final $(s i \hat{)}$ se produce en una palabra oxítona, obviamente, no existe una sílaba postónica. El primitivo fonológico es $\mathrm{L}+\mathrm{H}^{*}$. La asociación fonológica primaria vincula el tono a la sílaba acentuada, en el nivel jerárquico de esta sílaba acentuada. La asociación fonológica secundaria vincula ese tono con el acento de frase, en un nivel jarárquico superior. La sílaba acentuada se incrusta en 
la inflexión ascendente del acento de frase. Se observa la diferencia entre una ip continuativa (este caso) y una ip conclusiva (en la Fig. 5), los ítems son equivalentes, difieren en la lectura pragmática: la marca continuativa y la marca conclusiva. Estebas Vilaplana (2006) explica el comportamiento tonal de los acentos tonales dentro de palabras oxítonas por un fenómeno de truncamiento. El pico tonal se actualiza en la sílaba acentuada por ausencia de espacio para culminar en la postónica, inexistente. En los acentos tonales en palabras proparoxítonas y paroxítonas, el espacio postónico permite la culminación del pico tonal fuera del espacio de la sílaba acentuada. Habría que indicar que en este dialecto se producen acentos con el pico en la sílaba acentuada y otros con la culminación en la sílaba postónica. Sin embargo, es la presencia del acento de frase H- la que influye en la diferencia de asociación de acentos tonales en palabras oxítonas y de acentos tonales en palabras proparoxítonas y paroxítonas. El último acento tonal final (espantarlos) es L*. Este acento tonal se comunica paradigmáticamente con el nivel bajo (L) del informante (Ladd 1996: 91; Beckman et al. 2005). Esta ip concluye con el acento de frase (L-) y con el tono de frontera final de IP (L\%). Esta última frase entonativa (conclusiva) está en contraste con las frases entonativas intermedias, continuativas.

Figura 6

Fragmento de tres ip y la IP, acento tonal final, acento de frase, tono de frontera

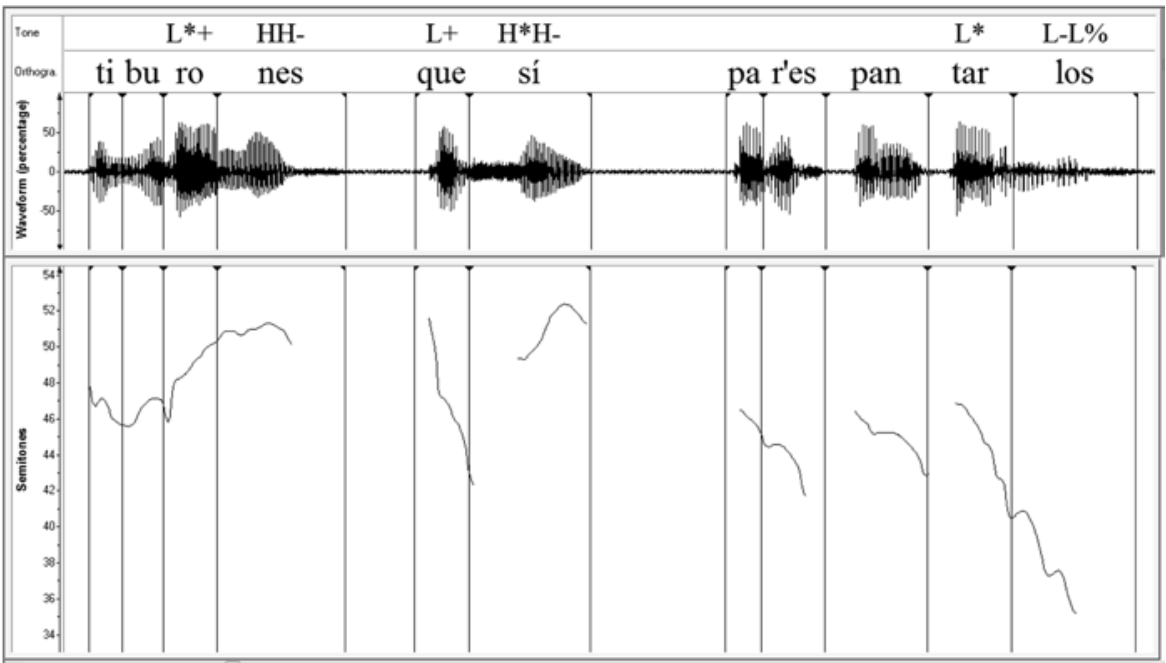

En la Fig. 7 se presentan dos acentos tonales y un acento tonal final de ip. Pertenecen a esta oración: [(Le preguntó si estaba dispuesto a hacerlo por el mismo jornal que le pagaban los pescadores por ayudarlos a pescar) ip, (y Euclides le dijo que sí,) ip (pero con un recargo de cinco reales los domingos) ip IP]. Los dos acentos tonales iniciales (pescadores, ayudarlos) siguen el esquema panhispánico. Tienen un valle en el inicio de la sílaba acentuada, se desarrollan en la sílaba acentuada y culminan en la sílaba postónica. Son los acentos tonales estándares en el pretonema (Sosa 1999: 187-195; Face 2001; Hualde 2003; Face y Prieto 2007). El acento tonal final se actualiza en una palabra oxítona. 
Figura 7

Fragmento de una ip, acentos tonales, acento tonal final, acento de frase

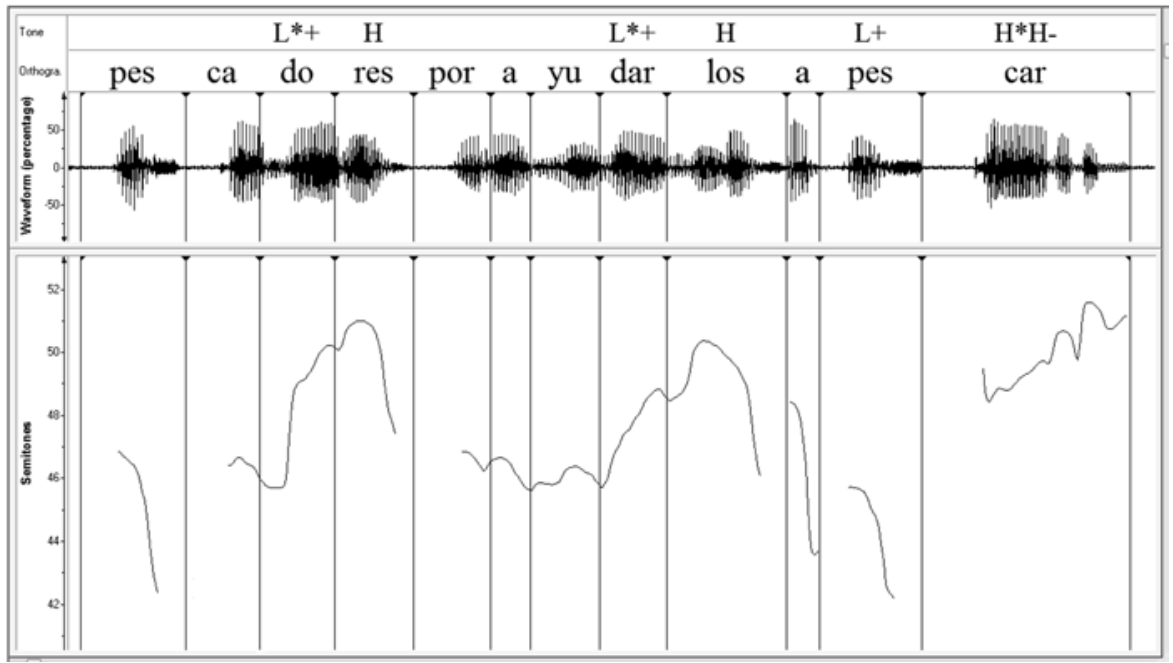

El valle comienza en el inicio de la sílaba acentuada y culmina en esa misma sílaba. De nuevo, las dos asociaciones (la primaria y la secundaria) se producen en la sílaba acentuada: la primaria vincula el tono con la sílaba acentuada, en el mismo nivel jerárquico y la secundaria vincula este tono con el acento de frase, en un nivel jerárquico superior en el fraseo entonativo. De nuevo, no es el efecto de truncamiento el que justifica la presencia del pico en acentos tonales que se realizan en palabras oxítonas (Estebas Vilaplana 2006; 2007), sino la posición del acento en el final de la palabra y la influencia de la inflexión creciente del acento de frase (H-), con la intención pragmática del informante de marcar la frase entonativa con un valor continuativo (Pierrehumbert e Hirschberg 1986; Pierrehumbert e Hirschberg 1990).

En la Fig. 8 se puede observar un fragmento de ip que es parte de una oración mayor, es ésta: [(Luego lo vio desaparecer en un matorral de corales,) ip (y justo cuando pensaba que no podía tener más aire oyó la voz a sus espaldas) ip IP]. El primero acento tonal (luego) presenta un valle en el inicio de la sílaba acentuada, el tono asciende y concluye en la sílaba acentuada y baja. Sube en la sílaba postonica y recupera un valor similar a la acentuada (ver la Fig. 1 y Hualde 2003). El segundo acento tonal (vio) realiza un recorrido tonal equivalente, excepto en la sílaba postónica, que se eleva considerablemente con respecto a la sílaba acentuada. La brevedad de la palabra monosílaba permite el crecimiento en la primera sílaba del ítem adyacente. Entonces, el primer acento tonal es $\mathrm{L}+\mathrm{H}^{*}$ y el segundo acento tonal es $\mathrm{L}^{*}+\mathrm{H}$. El tercer acento tonal se actualiza en una palabra oxítona (desaparecer). El acento tonal es $\mathrm{L}+\mathrm{H}^{*}$, con el pico tonal dentro del desarrollo temporal de la sílaba acentuada. El cuarto acento tonal también se actualiza dentro de una palabra oxítona. Los dos acentos (el tercero y el cuarto) son $\mathrm{L}+\mathrm{H}^{*}$. El pico tonal concluye dentro de la sílaba acentuada. $\mathrm{El}$ acento tonal final se produce en una palabra paroxítona. La estructura acentual permite que la sílaba postónica reciba la influencia del acento de frase, por ello, el acento tonal es $\mathrm{L}^{*}+\mathrm{H}$. La asociación primaria vincula el tono con la sílaba acentuada 
Figura 8

Fragmento de una ip, acentos tonales, acento tonal final, acento de frase

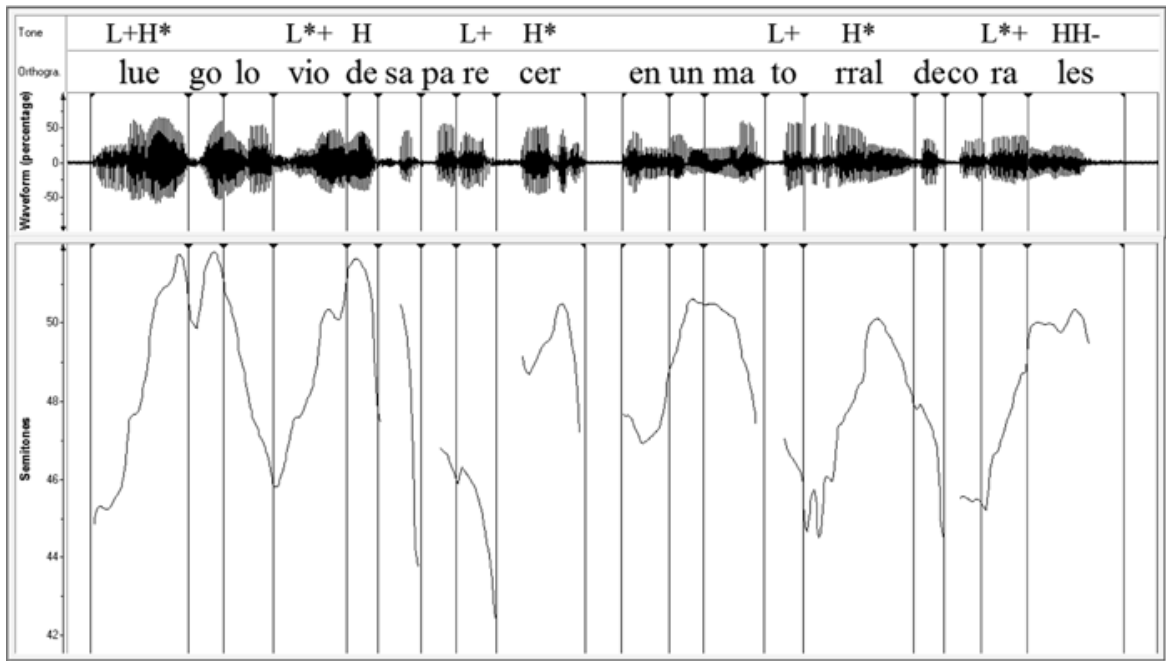

(L*), en el mismo nivel jerárquico, el de la sílaba acentuada. Además, la asociación fonológica secundaria vincula el tono con el acento de frase, en un nivel jerárquico superior, el del acento de frase y la frase entonativa intermedia. El tono periférico, en la sílaba postónica, se incrusta con la inflexión ascendente del acento de frase ( $\mathrm{H} \mathrm{H}-)$. El resultado es la marca de continuidad en este tipo de frase intermedia. Contrasta con las frases finales, la intermedia y la mayor, que tienen la marca de conclusión.

Figura 9

Fragmento de una ip, acentos tonales, acento tonal final, acento de frase

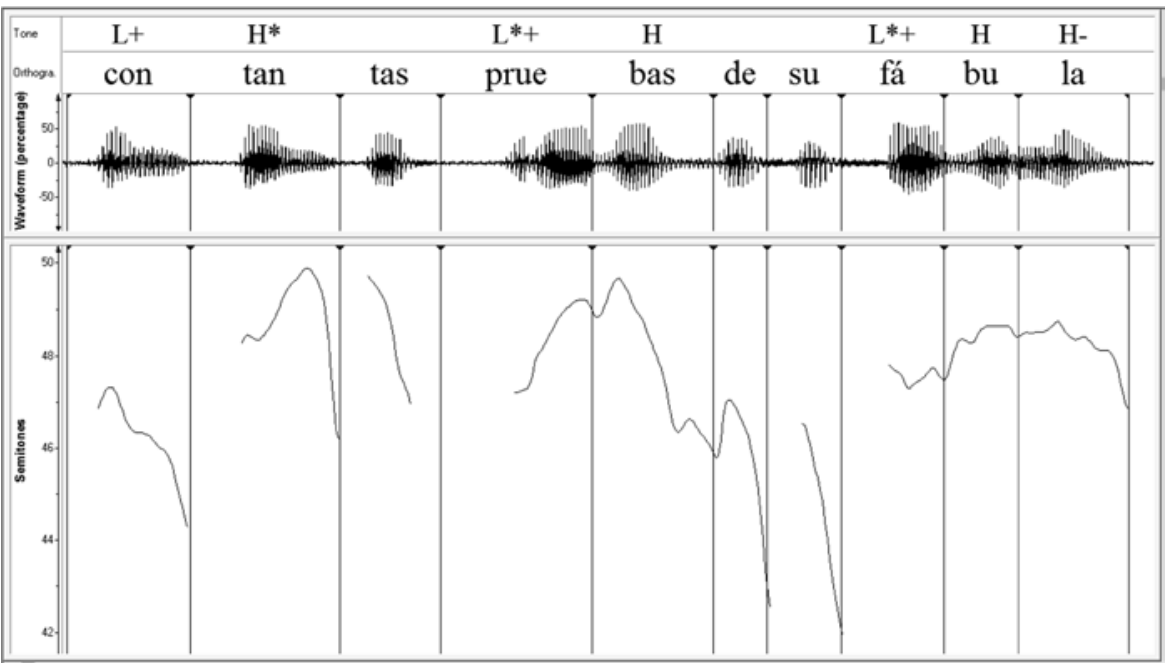


El fragmento de esta ip (en la Fig. 9) integra una oración más compleja, es ésta: [(Por esos días,) ip (Euclides había salido a flote con tantas pruebas de su fábula,) ip (que ya no era asunto de seguir triscando aretes y anillos desperdigados entre los corales,) ip (sino de capitalizar una empresa grande para rescatar el medio centenar de naves con la fortuna babilónica que llevaban dentro.) ip IP]. El primer acento tonal es $\mathrm{L}+\mathrm{H}^{*}$, con una realización tonal similar a los ejemplos ya explicados: un valle en el inicio de la sílaba acentuada y un pico que concluye dentro de esa sílaba acentuada (ver Fig. 1 y Hualde 2003). No es un acento tonal estándar para el español panhispánico. El segundo acento tonal (pruebas) es $\mathrm{L}^{*}+\mathrm{H}$, el pico tonal supera los límites de la sílaba acentuada y se despliega sobre la sílaba postónica. Este acento tonal sí es típico del español panhispánico (Sosa, 1999: 187-1995); no lo es en Sosa (1991). En general, es el acento tonal que aparece en los acentos prenucleares, en emisiones de foco ancho; contrasta con el acento nuclear y con el acento tonal en el foco estrecho (Face 2001; Face y Prieto 2007). En este dialecto de Colombia, por lo menos en este informante, fluctúan los dos tipos de acento. El acento tonal final se produce dentro de una palabra proparoxítona. El desarrollo tonal es la sílaba acentuada, el tono periférico en la primera sílaba postónica y una segunda sílaba postónica. Según Estebas Vilaplana $(2006 ; 2007)$ es en estos acentos tonales, producidos en palabras proparoxítonas, en que el espacio tonal es mayor y la posibilidad de despliegue del pico tonal se amplía hacia la derecha. El acento tonal final (fábula) es $\mathrm{L}^{*}+\mathrm{H}$, el tono es bajo en la sílaba acentuada y concluye en la sílaba postónica, el tono periférico. La inflexión ascendente del acento de frase influye en el acento tonal final. El tono se vincula con la sílaba acentuada en una asociación primaria, ese tono, a su vez, se incrusta en el acento de frase en una asociación fonológica secundaria, en un nivel superior de la jerarquía, en el nivel de la ip y de su acento de frase. La fuerza ascendente de la frase intermedia influye en la asociación fonológica de este acento tonal. Las frases intermedias tienen la marca de continuidad (H-) y contrastan con la frase intermedia y mayor final (L- L\%), con la marca de conclusión.

Este fragmento (en la Fig. 10) pertenece a esta oración: [(El aire era tan tenue,) ip y el mar era tan sereno y diáfano,) ip (que Florentino Ariza se sintió como si fuera su propio reflejo en el agua.) ip IP]. El primer acento tonal (mar) se produce en una palabra oxítona. El acento tonal es $\mathrm{L}+\mathrm{H}^{*}$, con un valle en el incio de la sílaba acentuada y un pico que concluye en esa sílaba acentuada. No es un tipo de acento tonal estándar para el español panhispánico. El segundo acento tonal presenta un valle en la acentuada y un pico que concluye fuera de la esa sílaba acentuada, el acento es $\mathrm{L}^{*}+\mathrm{H}$, este acento tonal sí es estándar en el español (Sosa 1999; Face 2001; Hualde 2003; Face y Prieto 2007). El tercer acento tonal (sereno) es similar al acento tonal anterior, es $\mathrm{L}^{*}+\mathrm{H}$. Por último, el acento tonal final es $\mathrm{L}+\mathrm{H}^{*}$, recibe la influencia tonal del acento de frase (H-), que mantiene las tres sílabas en un nivel similar de altura tonal. La frase entonativa intermedia tiene la marca de continuidad y contrasta con el acento de frase y el tono de frontera final (L- L\%), que tiene la marca de conclusión. 
Figura 10

Fragmento de ip, acentos tonales, acento tonal final, acento de frase

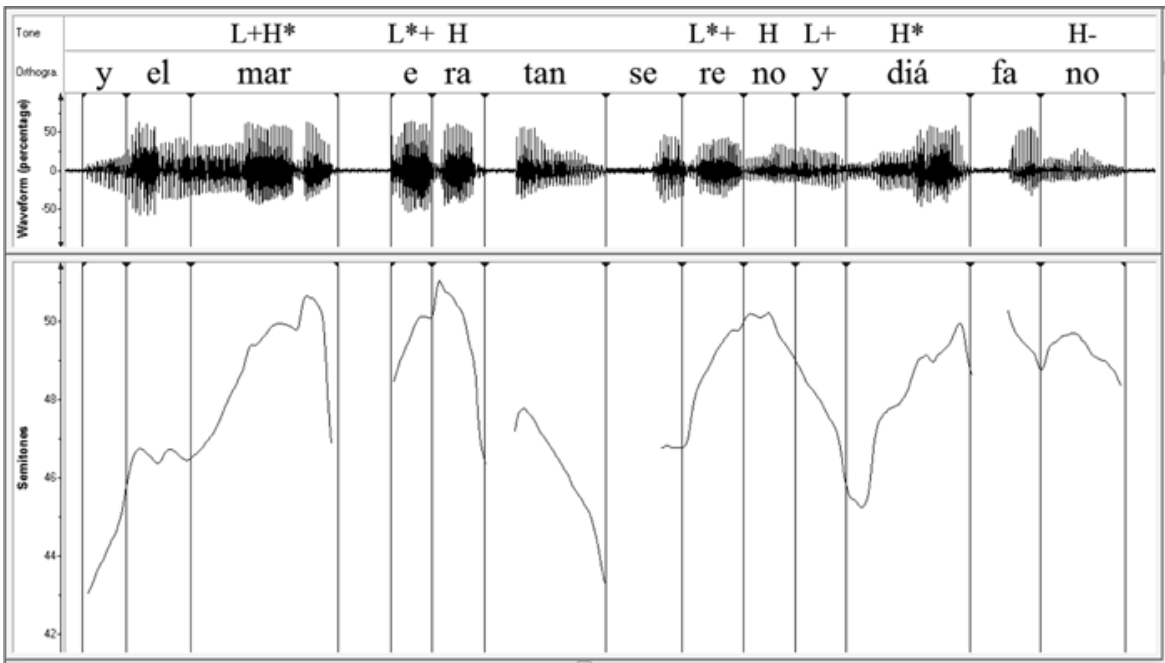

\section{CONCLUSIÓN Y DISCUSIÓN}

4.1. Acentos tonales no finales. Los acentos tonales no finales se apartan de la taxonomía estándar para el español panhispánico. El pretonema no presenta un tipo de acento único $\mathrm{L}^{*}+\mathrm{H}$, característico de la mayoría de los dialectos (Sosa 1999; Face 2001, Face y Prieto 2007). Sin embargo, el dialecto de Buenos Aires (Toledo 2000; Colantoni y Gurlekian 2004; Colantoni 2005) y el dialecto de Santiago de Chile (Ortiz-Lira 2003) presentan pretonemas con acentos tonales $\mathrm{L}+\mathrm{H}^{*}$ y $\mathrm{H}^{*}+\mathrm{L}$, es decir, con el pico tonal dentro del límite de la sílaba acentuada. Sosa (1991) también presenta acentos tonales en el español bogotano con el pico dentro de los límites de la sílaba acentuada, sobre todo en emisiones interrogativas absolutas de este dialecto. Asimismo, Toledo (2006 a) encuentra acentos tonales con el pico tonal dentro de la sílaba acentuada, en el dialecto de Madrid, en microdiscursos.

4.2. Acentos tonales finales. Los acentos tonales finales están influidos por la posición del acento en las palabras que contienen estos acentos tonales. Esa influencia es ejercida también por el acento de frase por medio de una asociación secundaria, el tono se vincula con esos acentos de frase intermedia (H-). En los acentos tonales dentro de palabras oxítonas, la sílaba acentuada se incrusta en la inflexión ascendente de ese acento de frase $\left(\mathrm{L}+\mathrm{H}^{*}\right)$. En los acentos tonales en palabras paroxítonas, es el tono periférico el que se vincula con la inflexión ascendente del acento de frase $\left(\mathrm{L}^{*}+\mathrm{H}\right)$. En los acentos tonales en palabras proparoxítonas, el tono periférico (la primera sílaba postónica) se incrusta en la inflexión ascendente del acento de frase. Sin embargo, pueden producirse acentos con el pico tonal en la sílaba acentuada o picos con picos en la sílaba postónica. La lectura de este tipo de acento es más coherente en habla de laboratorio (Toledo y Ramírez Verdugo 2007; Toledo, enviado), que en textos leídos y discursos (Toledo 2006 b). 
4.3. Acentos de frase, tonos de frontera y contraste fonológico. Las frases entonativas intermedias presentan una marca de continuidad textual o discursiva. Es la asociación fonológica secundaria la que permite la recuperación del contraste y la marca de continuidad. Los tonos (en la sílaba acentuada o en el tono periférico) se incrustan en el acento de frase, de esta manera están marcados y comunican la diferencia pragmática de continuidad. Los acentos tonales finales y el acento de frase se perciben todos como acentos altos. Así, se confrontan con acentos tonales sumados a acentos de frase y tonos de frontera bajos, con la marca de conclusión textual o discursiva. En suma, los tonemas $\mathrm{L}^{*}+\mathrm{H} \mathrm{H}-\mathrm{y} \mathrm{L}+\mathrm{H}^{*} \mathrm{H}-$ (con la marca de continuidad) contrastan con los tonemas L* L- L\% y L+H* L- L\% (con la marca de conclusión).

4.4. Comparación final. Los resultados encontrados en este trabajo son similares a los hallazgos obtenidos en habla de laboratorio y en habla semiespontánea, en otros dialectos del español. En habla de laboratorio, Gurlekian y Toledo (en prensa) estudian emisiones declarativas constituidas por dos acentos tonales, el acento nuclear y los tonos de frontera final ( $\mathrm{SN}+\mathrm{SV}+\mathrm{SPrep})$. El análisis es parte del Amper-Argentina (Atlas Multimedia de la Prosodia del Espacio Románico, en Argentina). En esos resultados se observa una reestructuración de las emisiones constituidas por una frase intermedia (SN ip) y una frase intermedia y final (SV + SPrep ip IP). Los acentos tonales ( $\mathrm{SN}$ ip) se producen dentro de los tres tipos de palabras: paroxítonas, proparoxítonas y oxítonas. Se observan mecanismos fonológicos similares a los presentados en este dialecto colombiano. De manera similar, Román M. y Cofré V. (en prensa) presentan resultados similares dentro del marco del Amper-Chile. La reestructuración de las oraciones declarativas muestran una frase intermedia en el SN. Este SN tiene un acento tonal dentro de palabras paroxítonas, proparoxítonas y oxítonas. Los mecanismos fonológicos parecen equivalentes, aunque no están explicitados directamente. Asimismo, (Zamora Salamanca et al, 2005) lo registran en Castilla y León, en las ciudades de Salamanca y Valladolid (Amper-CyL). Del mismo modo, Ramírez Verdugo (2005) lo muestra en los corpus de Madrid (Amper-MAD). Tampoco en estos dos últimos estudios se explicita de manera directa, se desprende de los datos presentados y de los contornos entonativos analizados. Los resultados en el corpus de Colombia y estudiado en este trabajo también son similares a los propuestos en habla semiespontánea. Toledo (2006 b) lo observa en español de Buenos Aires: (1) una implementación fonética más superficial que en la formalizacion de los acentos tonales para dar cuenta de las diferencias provocadas por el acento léxico en posiciones diferentes (paroxítonas, proparoxítonas y oxítonas), (2) una asociación fonológica primaria y una posterior asociación fonológica secundaria entre el tono y los acentos de frase y de frontera final, (3) un contraste lingüístico entre la frontera intermedia (ip) y la frontera final (ip IP), (4) un contraste semántico y pragmático entre ambas fronteras, esto es, una marca de continuidad opuesto una de conclusión. De manera similar, Toledo (2007) registra mecanismos fonológicos equivalentes en microdiscursos de español madrileño. En suma, parece que la frase entonativa intermedia y su acento de frase es parte del fraseo entonativo del español, tal como se sugiere en estudios anteriores de Nibert (2000) y de Hualde (2003), entre otros. 


\section{OBRAS CITADAS}

Alvord, S. 2006. Spanish Intonation in Contact: the Case of Miami Cuban Bilinguals. Tesis doctoral. Minnesota: University of Minnesota.

Barjam, J. 2004. The Intonational Phonology of Porteño Spanish. Tesis de maestría. Los Angeles: University of California at Los Angeles.

Beckman, M., M. Díaz-Campos, J. Tevis Mc Gory y T. Morgan. 2002. "Intonation across Spanish, in the tones and break indices framework". Probus 14. 9-36.

—. J. Hirschberg y S. Shattuck-Hufnagel. 2005. "The original ToBI system and the evolution of the ToBI framework". Ed. S. Jun. En línea: www.ling.ohio-state.edu/ tobi/ JunBook /Beck HirschShattuckToBI.pdf.

Colantoni, L. 2005. "Peak Alignment of Pre-nuclear and Nuclear Accents in Argentine Spanish", $2^{\text {nd }}$ Spanish ToBI Workshop. 22 de junio de 2005. En línea: www. seneca.uab.es/ filologia catalana /papi/tobi/Colantoni.ppt.

—_ y Gurlekian, J. 2004. "Early Peak Alignment and Deep Fall in Buenos Aires Broad Focus Declaratives". 9th Conference on Laboratory Phonology. Department of Linguistics, University of Illinois at Urbana-Champaign. 24-2 de junio de 2004. En línea: www.linguist. jussieu.fr / marandin /pdf/ cours2.pdf.

Estebas Vilaplana, E. 2006. "Word edge tones in Spanish prenuclear accents". Estudios de Fonética Experimental XV. 11-42.

2007. "The phonological status of English and Spanish prenuclear F0 peaks". Atlantis 29, 2. 39-57.

Face, T. 2001. Intonational Marking of Contrastive Focus in Madrid Spanish. Tesis doctoral. Ohio: Ohio State University.

— y Prieto, P. 2007. "Rising accents in Castilian Spanish: a revision of Sp-ToBI". Journal of Portuguese Linguistics 5,2/6,1. 91-115.

Grice, M. 1995. The Intonation of Palermo Italian: Implications for Intonation Theory. Tübingen: Niemeyer.

Gurlekian, J. y G. Toledo. en prensa. "Datos preliminares del Amper-Argentina: las oraciones declarativas e interrogativas absolutas sin expansión". Actas del IV Congreso de Fonética Experimental. Granada. 11-14 de febrero de 2008.

Gussenhoven, C. 2002. "Phonology of intonation. State-of-the-article". Glot International 6. 271-284.

Heusinger, K von. 2007. "Discourse Structure and Intonational Phrasing". Topic and Focus: Intonation and Meaning. Theoretical and Crosslinguistic Perspectives. Eds. D. Büring, M. Gordon y Ch. Lee. Dordrecht: Kluwer. 265-290.

Hualde, J. 2003. "El modelo métrico y autosegmental". Teorías de la entonación. Ed. P. Prieto. Barcelona: Ariel. 155-184.

Jun, S. 2005. "Prosodic typology". Prosodic Typology: The Phonology of Intonation and Phrasing. Ed. S. Jun. Reino Unido y Europa: Oxford University Press. 430-457.

Ladd, R. 1996. Intonational Phonology. Cambridge: Cambridge University Press.

Nibert, H. 2000. Phonetic and Phonological Evidence for Intermediate Phrasing in Spanish Intonation. Tesis doctoral. Urbana-Champaign, Illinois: University of Illinois at UrbanaChampaign.

Ortiz-Lira, H. 2003. "Los acentos tonales en un corpus de español de Santiago de Chile: su distribución y realización", La tonía: dimensiones fonéticas y fonológicas . Eds. E. Herrera Z. y P. Martín Butragueño. México: El Colegio de México. 303-316.

Pierrehumbert, J. 1980. The Phonology and Phonetics of English Intonation. Tesis doctoral. Cambridge: Massachusetts Institute of Technology. 
y J. Hirschberg 1986. "The intonational structuring of discourse". Proceedings of the $24^{\text {th }}$ Annual Meeting of the Association for Computational Linguistics. En línea: citeseer. ist.psu.edu/julia86intonational.html.

— y M. Beckman. 1988. Japanese Tone Structure. Cambridge: The MIT Press.

- y J. Hirschberg. 1990. "The meaning of intonational contours in the interpretation of discourse". Intentions in Communication. Eds. P. Cohen, J. Morgan y M. Pollock. Cambridge: the MIT Press. 271-312.

Prevost, S. 1995. A Semantic of Contrast and Information Structure for Specifying Intonation in Spoken Language Generation. Tesis doctoral. Pensilvania: University of Pennsylvania.

Ramírez Verdugo, M. 2005. "Aproximación a la prosodia del habla de Madrid", Estudios de Fonética Experimental XIV. 309-326.

Roman M., D. y V. Cofré V. en prensa. "Rasgos prosódicos de oraciones sin expansión, del español de Santiago de Chile en habla femenina”, Actas del IV Congreso de Fonética Experimental. Granada. 11-14 de febrero de 2008.

Sosa, J. 1991. Fonética y fonología de la entonación del español hispanoamericano. Tesis doctoral. Massachusetts: University of Massachusetts.

- 1999. La entonación del español: Su estructura fónica, variabilidad y dialectología. Madrid: Cátedra.

Toledo, G. 2000. "H en el español de Buenos Aires". Langues et Linguistique 26. 107-127.

_. 2006 a. "Acentos estrellados: una argumentación". Estudios de Fonética Experimental XV. 99-131.

—. 2006 b. "Fonología entonativa en discursos de Buenos Aires: Asociación fonológica secundaria de T* frente a H-'. Language Design. Journal of Theoretical and Experimental Linguistics 8. 131-152.

- 2007. "Fraseo en español peninsular y modelo autosegmental y métrico". Estudios Filológicos 42. 227-243.

—. enviado. "Fonología de la entonación: asociación primaria y secundaria en dialectos antípodas". Revista Española de Lingüística.

- y M. Ramírez Verdugo. 2007. "Spanish phrasing: Secondary association of T* in face of intermediate phrase edge tone H- (laboratory data)". Phonetics and Phonology in Iberia (PaPI) 2007. Braga, Portugal (en proceso).

Zamora Salamanca, F., M. Carrera de la Red y M. Meléndez Matías. 2005. "Variación geoprosódica en dos entonaciones de Castilla y León: Análisis de frases declarativas e interrogativas sin expansión". Estudios de Fonética Experimental XIV. 125-139. 